\title{
The Application of High Resolution Melting in the Analysis of Simple Sequence Repeat Markers for Fingerprinting of Brinjal (Solanum melongena L.) Hybrid and its Parental Lines
}

\author{
Tejaswini S Ghatage ${ }^{1}$, Pulak Das ${ }^{1}$ \\ ${ }^{1}$ Adithya Biotech lab \& Research Pvt Ltd, Raipur, 492001, Chhattisgarh, India
}

\begin{abstract}
Eggplant (Solanum melongena) is recognized as one of the most important members of the Solanaceae family. Knowledge of brinjal genome organization is rather limited compared to other Solanaceous crops, especially tomato and potato. It is essential to develop an efficient method to build the fingerprinting database of commercial brinjal hybrids and their parental lines for seed purity analysis. Use of morphological differences, between true hybrids and off types in grow out test (GOT) for genetic purity analysis, are not always apparent and cannot be recognised easily. Further, morphological traits are costly, tedious to score and environment sensitive. Alternatively, it is suggested that recent breakthrough in molecular markers can be employed in genetic purity analysis. The objective of this investigation were to fingerprint commercial brinjal hybrid and their parental lines using SSR molecular markers to develop an efficient fingerprinting method for seed purity analysis in agriculture production. Brinjal $F_{l}$ hybrid $555 \times R J$ and it's parents 555 as female and RJ as male were studied for identification and genetic purity testing with high resolution melt analysis and agarose gel electrophoresis. There are 48 brinjal microsatellite loci i.e. SSR primers were used for the analysis. Microsatellite loci, P22 were found to be heteroallelic for parents. P22 identified female specific 180bp repeat and male specific 190bp repeat. However, hybrid $(555 \times R J)$ exhibited the alleles of both parents, confirming the heterozygosity of the hybrid with the presence of two bands at 180bp and 190bp. High resolution melt analysis shows three different melting curves for male, female and hybrid. The result suggested that agarose gel electrophoresis and high resolution melting curve analysis could be used as potential, efficient and valuable methods for genetic purity analysis of brinjal hybrid and parental lines, and high resolution melting curve analysis should be given priority compared with agarose gel electrophoresis for its high accuracy and high efficiency.
\end{abstract}

Keywords: Agarose gel electrophoresis; Brinjal; Genetic purity; High resolution melting curve analysis; Solanum melongena

\section{Introduction}

Brinjal (Solanum melongenaL., $2 \mathrm{n}=2 \mathrm{x}=24$.), also known as aubergine or eggplant, belongs to the family Solanaceae, but unlike most of the solanaceous crop species, it is endemic to the old world. Its progenitor is presumed to have been the African species $S$. incanum ${ }^{1}$, but its centre of domestication and genetic diversity lies in the Indo-Burma region, where it has been grown for at least 1,500 years ${ }^{2}$. The production of brinjal is highly concentrated, in five countries, namely China, India, Egypt, Iran and Turkey with 90 percent of output coming from them. India is the second (25\%) largest producer of brinjal in the world (Annonymous, 2014). Eggplant is the third most important solanaceous crop worldwide after potato and tomato ${ }^{3-4}$.

Evaluation of genetic resources is crucial for breeders to develop new cultivars or for further improvement of the existing ones in response to changes in consumer demand. Molecular markers have enormous potential to explore genetic diversity by detecting polymorphisms, and are useful tools for breeding, genotype identification, and determination of genome organization and evolution in plants ${ }^{5}$. Microsatellites (SSR) markers are a popular source of genetic markers owing to their high reproducibility, multi-allelic nature, co-dominant inheritance, abundance, and wide genome coverage. High level of polymorphism makes SSR an ideal marker for mapping and diversity studies, fingerprinting and population genetics (Nunome et al., 2003) . A $^{6}$ number of SSR markers have been identified in Solanaceae, (eggplant inclusive) (Bindler et al., 2007) ${ }^{7}$. Shorter SSR motifs and longer SSRs tended to be associated with a greater number of alleles. Several workers have contributed to the characterization of the largest genus of Solanaceae family (Bohs, 1999) ${ }^{8}$. Few studies had been performed to determine genetic diversity of eggplant using random amplified polymorphic DNA (RAPD) (Nunome et al., 2001) ${ }^{9}$, amplified fragment length polymorphisms (AFLP) (Mace et al., 1999) ${ }^{10}$, simple sequence repeats (SSR) (Prohens et al., 2012) ${ }^{11}$, and inter simple sequence repeat (ISSR) (Isshiki et al., 2003) ${ }^{12}$.

High resolution melting(HRM) curve analysis is a novel, closed-tube, post-PCR technique invented in 2003 (Wittweret al., 2003) ) $^{13}$ to detect DNA variation, which has been used in clinical chemistry, epidemical analysis, microorganism typing and molecular biology (Wu et al., $2008^{14}$; Hofingeret al., 2009 ${ }^{15}$; Ganopouloset 
al., 2011 ${ }^{16}$; Thomsen et al., 2012 ${ }^{17}$ ). In HRM experiments, the target sequence is amplified by PCR in the presence of a saturating fluorescent dye (e.g. LCGreen ${ }^{\circledR}$, EvaGreen $\left.{ }^{\circledR}\right)$. HRM dye fluoresces strongly only when bound to dsDNA. This change of fluorescence during an experiment can be used both to measure the increase in DNA concentration during PCR amplification and, subsequently, to measure temperature-induced DNA dissociation during high resolution melting. After PCR in the presence of the dsDNA-binding fluorescent dye, amplifications are briefly denatured and then rapidly reannealed. If the DNA sample is heterozygous, perfectly matched hybrids (homoduplexes) and mismatched hybrids (heteroduplexes) are formed. When the temperature is slowly increased again, the dsDNA begins to melt, and the shapes of melting curves are significantly different based on the G-C content, length and sequence of the fragments (Herrmannet al., 2006) ${ }^{18}$. In fact, the HRM technique is so sensitive that it can even detect single base variations between homozygous samples. SSR-HRM method has the advantages of high efficiency and high accuracy, advantages of high efficiency and high accuracy, which has been successfully used for variety identification of grape and olive (Mackay et al., 2008) ${ }^{19}$, common bean (Ganopoulos et al., 2012), sweet cherry (Ganopoulos et al., 2012) ${ }^{20}$, rice (Zhu et al., 2013) ${ }^{21}$, and cotton (Tejaswini et al., 2016) ${ }^{22}$.

\subsection{Plant material}

\section{Materials and methods}

Leaf sample of commercial hybrid $(555 \times \mathrm{RJ})$ and their parental lines (Table 1) used in this study were acquired from Aditya Seeds Pvt Ltd, Raipur. Fresh young leaves from all plants were collected for DNA extraction.

\subsection{DNA extraction}

Genomic DNA was isolated according to a modified CTAB method (Zhu et al., 2010) ${ }^{23}$. The concentration and quality of the obtained genomic DNA samples were estimated by measuring O.D. at 260/280 $\mathrm{nm}$ in UV spectrophotometer. Finally, all the genomic DNA samples were diluted to a final concentration of 40ng/ $\mu \mathrm{l}$ with $1 \mathrm{X}$ TE buffer (10mM Tris-HCL; pH 8.0; 1mM EDTA). Intactness of genomic DNA was checked by agarose gel electrophoresis. DNA samples were stored at $-20^{\circ} \mathrm{C}$ for further use.

\subsection{SSR-PCR amplification and agarose gel electrophoresis analysis}

SSR amplification was conducted in a $20 \mu \mathrm{l}$ volume containing $40 \mathrm{ng}$ of genomic DNA, 10X buffer, $1 \mathrm{U}$ Taq DNA polymerase, $1.5 \mathrm{mM} \mathrm{MgCl}_{2}, 2.5 \mathrm{mM}$ dNTPs, 3pmol forward and 3pmol reverse primer. The PCR protocol consisted of an initial denaturation at $94^{\circ} \mathrm{C}$ for $5 \mathrm{~min}$, followed by 35 cycles of $94^{\circ} \mathrm{C}$ for $20 \mathrm{sec}$, annealing for $30 \mathrm{sec}$ at $58^{\circ} \mathrm{C}, 72^{\circ} \mathrm{C}$ for $1 \mathrm{~min}$ and final extension step of $72^{\circ} \mathrm{C}$ for $5 \mathrm{~min}$. The amplification reaction was carried out in thermo cycler (Applied Biosystems). The PCR products were analysed on $2 \%$ agarose gel along with 100bp molecular weight marker and photographed under UV light using Bio-Rad gel documentation system.

\subsection{SSR-HRM (Simple sequence repeat-high resolution melting curve) analysis}

SSR-HRM amplification was conducted in a $20 \mu \mathrm{l}$ volume containing $20 \mathrm{ng}$ of genomic DNA, 10X buffer, $1 \mathrm{U}$ Taq DNA polymerase, $1.5 \mathrm{mM} \mathrm{MgCl}$, 2.5mM dNTPs, 3pmol forward, 3pmol reverse primer, 20X EvaGreen dye (Invitrogen) and made up to $20 \mu \mathrm{l}$ with deionized water. The HRM amplification reaction procedure and melting analysis were performed as follows: $5 \mathrm{~min}$ initial denaturation at $95^{\circ} \mathrm{C}$ followed by 40 cycles of denaturation at $95^{\circ} \mathrm{C}$ for $20 \mathrm{sec}$, annealing at $58^{\circ} \mathrm{C}$ for $30 \mathrm{sec}$ and extension at $72^{\circ} \mathrm{C}$ for $1 \mathrm{~min}$. The amplification procedure was immediately followed by the high resolution melting steps: $90^{\circ} \mathrm{C}$ for 2 min and then the temperature was raised to $79^{\circ} \mathrm{C}$ to $83^{\circ} \mathrm{C}$, raised by $0.2^{\circ}$ each step and wait for $3 \mathrm{sec}$ for each step afterwards. The HRM curve was acquired and analysed on the AriaMX real time PCR system (Agilent technologies). After the verification of amplification curves and the presence of a specific melting peak for the microsatellite amplification, the melting curve stage was analysed.

\section{analysis by agarose gel el}

\section{Results}

Microsatellite markers were used for identification of brinjal hybrid $555 \times \mathrm{RJ}$ and it's parents 555 (female) and RJ (male) shown in (Table 1). 48 SSR primer paires were initially screened against hybrid $555 \times \mathrm{RJ}$ and it's parental lines. All of the primer pairs had good amplification. Out of 48 microsatellite primers, 6 pairs i.e. P162, P647, P451, P22, P83, P36, were scorable on agarose gel and showed polymorphism in parents (Table 2). Out of these P22 (Forward5' GGCGATACCATTGATGAACC 3' Reverse5' TTCCTGCTAGCAT CCTTCGT3') were used to identify the hybrid $555 \times$ RJ and it's parents. Primer P22 amplified two repeats of different lengths, out of which a repeats of $180 \mathrm{bp}$ was 555(female) specific and a repeat of 190bp was RJ (male) specific, were amplified in hybrid 555×RJ (fig 1). Micosatellite analysis has been successfully employed for parentage verification, hybrid identification, cultivar characterization and purity testing in other crop plants ${ }^{24-26}$. In the present study, microsatellite primer P22 strongly supports the hybridity. 


\subsection{SSR fragments analysis by high resolution melting curve analysis (SSR-HRM)}

Based on the results of agarose gel electrophoresis primer P22 were selected for high resolution melting curve analysis of hybrid $555 \times \mathrm{RJ}$ and it's parental lines. Materials having different amplification fragments were able to be identified by the shape of melting curves, which is the principle of HRM analysis. Significantly different curves were achieved based on the different amplifications of brinjal hybrid and it's parental lines (fig 2). Brinjal hybrid $(555 \times \mathrm{RJ})$ had two amplification fragments, (Red curves, and amplification sizes 180:190 bp). However, it's female line (555) had one amplification fragment, (Green curves, amplification size $180 \mathrm{bp}$ ), and male line (RJ) also had only one amplification fragment (Blue curves, amplification size $190 \mathrm{bp})$. As the results, hybrid $(555 \times \mathrm{RJ})$ and it's parental lines had significantly different curves, which could be used for hybrid purity. The present study indicates that Microsatellites banding and curve patterns of the parents compared with it's hybrid is able to clearly recognize the true hybrid and it's profile.

\section{Discussion}

Agarose gel electrophoresis was traditional method to analyze SSR fragments. In this study agarose gel electrophoresis was selected for initial analysis of the amplifications because of it's simple and affordable technology. The agarose gel electrophoresis results showed that SSR molecular markers were valuable for genetic analysis of brinjal because of it's high polymorphism. Based on the agarose gel electrophoresis results SSR primer, P22 which produce stable, distinct and polymorphic amplification was selected for high resolution melting curve analysis to compare their efficiency in seed purity analysis. However, high resolution melting curve analysis technique showed a potential application for plant genotyping. Compared with agarose gel electrophoresis, high resolution melting curve analysis have the advantages of high accuracy, high-throughout, high efficiency, and no-touch of toxic reagents. High resolution melting curve analysis was able to distinct the differences in $\mathrm{G}-\mathrm{C}$ content. The result of high resolution melting curve analysis was showed with significantly different curves based on different amplifications.

\section{Figures and Tables}

Table 1. Name of brinjal hybrid and their parental lines used in this study

\begin{tabular}{|l|l|l|}
\hline Hybrid & Female line & Male line \\
\hline $555 \times$ RJ & 555 & RJ \\
\hline
\end{tabular}

Table 2. Sequences of SSR primer pairs used in this study which shows polymorphism in parental lines.

\begin{tabular}{|c|l|l|}
\hline Primer Name & Forward Primer $\left(5^{\prime}{ }^{\prime}-3^{\prime}\right)$ & Reverse Primer (5'-3') \\
\hline P162 & GGACCGTTCAGGAGGTTACA & GCCATCATTCAACAAAACCGAAT \\
\hline P647 & CGGATTCGGTTGAGTCGATA & GTGCTTTGGTTCGGTCTTTC \\
\hline P451 & TGCATTGGTGGGCTAACATA & GCTCTTGACACAACCCCAAT \\
\hline P22 & GGCGATACCATTGATGAACC & TTCCTGCTAGCATCCTTCGT \\
\hline P83 & AAAGGCAGATTGCTGAAGGA & GATATGGCAGAGGCAATGCT \\
\hline & TCACGTCCACCATCCAACTA & GATCACGTGTGTGTGGGTTC \\
\hline
\end{tabular}

Fig 1

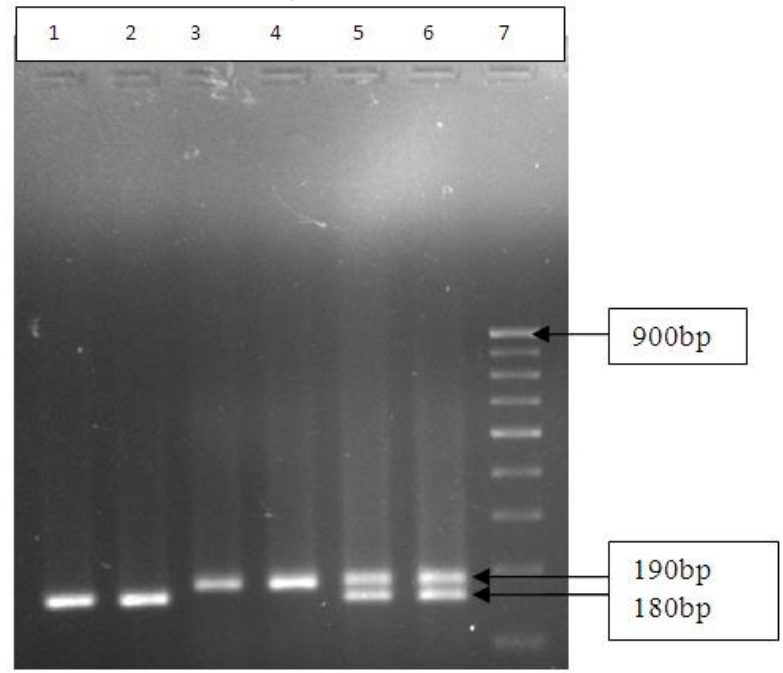

Fig 1. Amplification results of primer P22 from brinjal hybrid $555 \times \mathrm{RJ}$ and their parental lines. Lanes 1-2 represent female line555 (amplification size $180 \mathrm{bp}$ ), Lanes 3-4-represent male line RJ (amplification size 
190bp), Lanes 5-6- represent hybrid 555×RJ (amplification sizes 180:190 bp), Lane7-100bp ladder (100bp900bp).

Fig 2: Melt Difference Plots

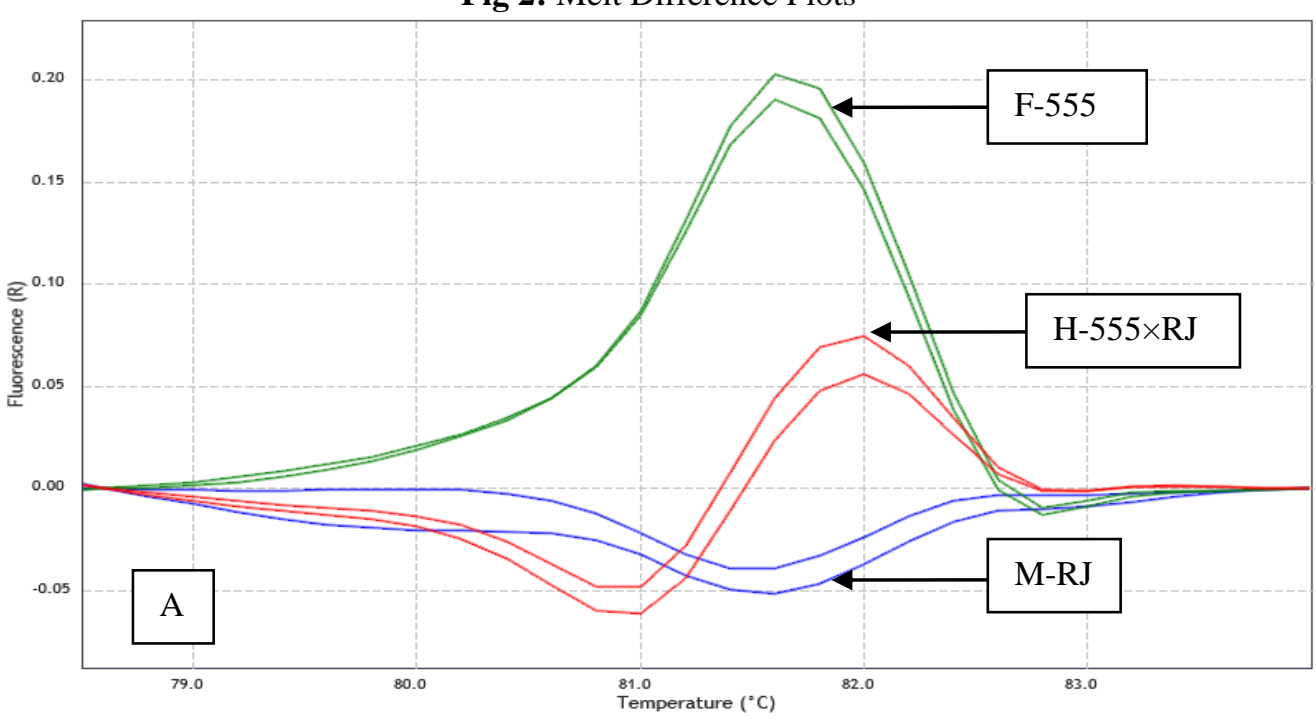

Melt Raw Derivative Curve

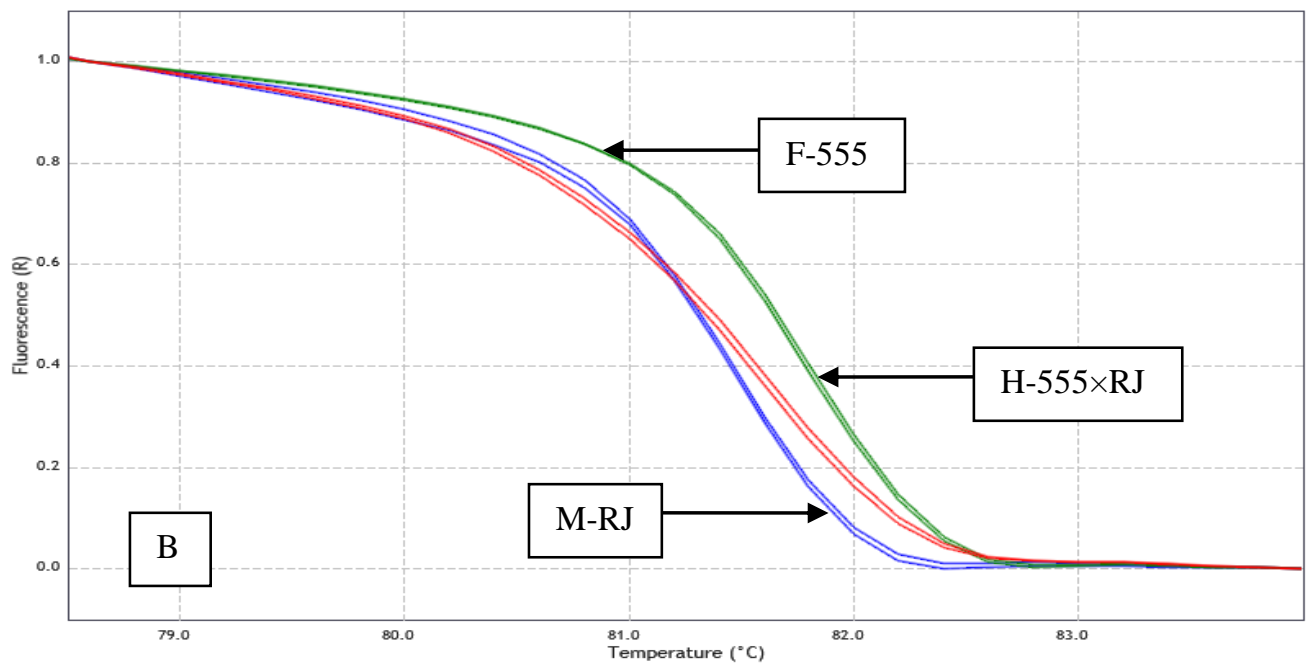

Fig 2. HRM analysis of amplification from $555 \times \mathrm{RJ}$ and their parental lines by P22. Melt difference plots (A) and Melt Raw Derivative Curve (B )for the identification of $555 \times \mathrm{RJ}$ (Red curves, amplification sizes 180:190 bp), 555 (Green curves, amplification size $180 \mathrm{bp}$ ) and RJ (Blue curves, amplification size 190bp) at SSR locus P22.

\section{Conclusion}

It is concluded from this study that it is possible to differentiate brinjal hybrid more accurately and efficiently from their parental lines using molecular markers. DNA markers are more accurate for determining hybrid seed purity. Hybrid purity was studied by agarose gel electrophoresis as well as high resolution melting curve analysis. Based on the result of this study, high resolution melting curve analysis is an efficient and potential method for genetic purity analysis of brinjal hybrid and their parental lines. Marker analysis will also result in considerable savings for the seed industry, as this technique may avoid the cost of storage for an entire season.

\section{Acknowledgments}

I would like to thanks Mr.Shailendra Jain,MD \& Ms Shubhika Jain,Director ABLRPL for providing me an opportunity to work in Adithya Biotech Lab \& Research Pvt Ltd.The help rendered by Dr. Indrani Chandra, Head department of Biotechnology, The University of Burdwan for her technical suggestion during the preparation of the manuscript is gratefully acknowledged. I am also thankful to Dr.A.K Verma, my Parents and all of them who helped me during this project. 


\section{References}

[1.] MC Daunay, RN Lester, G Ano ,Cultivated eggplants, Tropical plant breeding, Oxford university press, Oxford, United Kingdom(2001) .

[2.] A Stàgel , E Portis, L Toppino, GL Rotino, S Lanteri Gene based microsatellite development for mapping and phylogeny studies in eggplant,BMC Genomics( 9) (2008)357-370

[3.] FAOSTAT, Food and agricultural organization of united nations, USA(2010)

[4.] Manisha Mangal,Priti Upadhyay,Pritam Kalia,Characterization of cultivated and wild genotypes of brinjal(Solanum melongena L.) and confirmation of Hybridity using Microsatellite Markers.Vegetos (2016),29:2.

[5.] O T Adeniji, P Kusolwa, S O W M Reuben and P Deo, Molecular diversity among seven Solanum (eggplant and relatives) species assessed by simple sequence repeats (SSRs) markers. African Journal of Biotechnology Vol. 11(90), 8 November, 2012, pp. 1564315653

[6.] T Nunome, K Suwabe, A Ohyama, H Fukuoka Characterization of Tri nucleotide Microsatellites in Eggplant. Breed. Sci. (53) (2003) 77-83.

[7.] G Bindler, R van der Hoeven, I Gunduz, J Plieske ,A microsatellite marker based linkage map of tobacco. Theor. Appl. Genet. (114), (2007), 341-349.

[8.] I Bohs, Cyphomonda (Solanaceae) from Neotropica Monograph New York Botanical Garden. (1999) p. 63.

[9.] T Nunome, K Ishiguro, T Yoshida, M Hirai, Mapping of fruit shape and color development traits in eggplant (Solanum melongena L.) based on RAPD and AFLP markers. Breed. Sci.( 1), (2001) 19-26.

[10.] ES Mace, CG Gebhardt, RN Lester AFLP analysis of genetic relationships in the tribe Datureae (Solanaceae). Theor Appl. Genet.( 99) (1999),634-641.

[11.] J Prohens, A Rodriguez-Burruezo, M Dolores Raig'on, Nuez F ,Total phenolic concentration and browning susceptibility in a collection of different varietal types and hybrids of eggplant: Implications for breeding for higher nutritional quality and reduced browning, J. Am. Soc. Hort. Sci. 132(5), (2012) 638-646.

[12.] S Isshiki, S Suzuki, K Yamashita, RFLP analysis of mitochondrial DNA in eggplant and related Solanum species. Gen. Res. Crop Evol. (50) (2003) 133-137.

[13.] CT Wittwer, GH Reed, CN Gundry, JG Vandersteen, RJ Pryor,High-Resolution Genotyping by Amplicon Melting Analysis Using LCGreen. Clin Chem.49(6), (2003) 853-860.

[14.] Wu SB, M Wirthensohn, P Hunt, JP Gibson, M Sedgley, High resolution melting analysis of almond SNPs derived from ESTs. Theor Appl Genet.118(1), (2008) 1-14.

[15.] BJ Hofinger, HC Jing, KE Hammond-Kosack, K Kanyuka ,High-resolution melting analysis of cDNA-derived PCR amplicons for rapid and cost-effective identification of novel alleles in barley. Theor Appl Genet. 119(5), (2009) 851-869.

[16.] I Ganopoulos, A Argiriou, A Tsaftaris Microsatellite high resolution melting (SSR-HRM) analysis for authenticity testing of protected designation of origin (PDO) sweet cherry products. Food Control.22(3-4), (2011) 532-541.

[17.] N Thomsen, RG Ali, JN Ahmed, RM Arkell,High Resolution Melt Analysis (HRMA); a Viable Alternative to Agarose Gel Electrophoresis for Mouse Genotyping. PLoS ONE. 7(9): e(2012) 45252.

[18.] MG Herrmann, JD Durtschi, K Bromley, CT Wittwer, KV Voelkerding ,Amplicon DNA melting analysis for mutation scanning and genotyping: Cross-platform comparison of instruments and dyes, ClinChem.52(3), (2006) 494-503.

[19.] JF Mackay, CD Wright, RG Bonfiglioli, A new approach to varietal identification in plants by microsatellite high resolution melting analysis: application to the verification of grapevine andolive cultivars. Plant Methods.4(1): (2008) 8.

[20.] I Ganopoulos, I Bosmali, P Madesis, A Tsaftaris,Microsatellite genotyping with HRM (High Resolution Melting) analysis for identification of the PGI common bean variety Plake Megalosperma Prespon. Eur Food Res Technol.234(2012)501-508.

[21.] Yanfang Zhu, Qitian Wang, Jin Hu, Liwei Zhu, Jiancheng Wang, Shuijin Zhu and Yajing Guan, High resolution melting curve analysis: an efficient method for fingerprinting of hybrid rice cultivars and their parental lines. Australian journal of crop science 7(13) (2013) 2048-2053

[22.] Tejaswini S Ghatage, Pulak Das, High Resolution Melting Curve Analysis: an Efficient Method for Genetic Purity Analysis of Cotton (Gossypium hirsutum) Hybrid and their Parental Lines, IOSR Journal of Biotechnology and Biochemistry (IOSR-JBB) ISSN: 2455-264X, Volume 2, Issue 7 (Nov. - Dec. 2016), PP 49-53

[23.] YF Zhu, J Hu, Y Zhang, YJ Guan, XX Huang, SJ Zhu Transferability of SSR markers derived from cowpea (Vigna unguiculata L. Walp) in variety identification. Seed Sci Technol. 38 (2010) 730-740.

[24.] M Asif, M Rahman \& Y Zafar, Genotyping analysis of six maize (Zea mays L.) hybrids using DNA fingerprinting technology, Pak J Bot, 38 (2006) 1425-1430.

[25.] C H C M Bertini, I Schuster, T Sediyama, E G Barros \& M A Moreiral, Characterization and genetic diversity analysis of cotton cultivars using microsatellites, Genet Mol Bio, 29 (2006) 321-329.

[26.] N Tabbasam, M Rahman \& Y Zafar, DNA-based genotyping of sorghum hybrids, Pak J Bot, 38 (2006) $1599-1604$

[27.] Michał KNOPKIEWICZ, Magdalena GAWŁOWSKA and Wojciech ŚWIECICKI, The Application of High Resolution Melting in the Analysis of Simple Sequence Repeat and Single Nucleotide Polymorphism Markers in a Pea (Pisum sativum L.) Population,Czech J. Genet. Plant Breed., 50, 2014 (2) 151-156. 